\title{
DE LOS TRIBUNALES POPULARES A LAS COMISIONES DEPURADORAS. VIOLENCIA Y REPRESIÓN EN GUIPÚZCOA DURANTE LA GUERRA CIVIL Y EL PRIMER FRANQUISMO (1936-1945) $)^{1}$
}

Pedro Barruso Barés

Guipúzcoa, con una población de cerca de 300.000 personas en 1930, mantuvo un comportamiento político conservador a lo largo de la II República. La prueba más clara que demuestra esta afirmación fue la victoria electoral, en los sucesivos comicios celebrados en la etapa republicana, de las opciones conservadoras (coalición entre tradicionalistas y nacionalistas en 1931 y nacionalistas en 1933 y 1936). Por su parte, la izquierda logró buenos resultados en 1931 y 1936 logrando dos diputados en cada uno de los comicios. La izquierda, por su parte, sólidamente implantada en las principales localidades de la provincia como era el caso de San Sebastián, Irún y Éibar, que superaban los 10.000 habitantes y suponían el $40 \%$ de la población de Guipúzcoa, y en otras como Rentería, Mondragón o Pasajes entre las que superaban los 5.000 habitantes en $1930^{2}$. Del mismo modo la Guerra Civil fue breve en la provincia. A mediados de agosto de 1936 una parte importante de la provincia, la lindante con Navarra, estaba en manos de los sublevados que comenzaban su ofensiva sobre la frontera francesa. El día 4 de septiembre fue ocupado Irún y el 13 del mismo mes era ocupado San Sebastián. A finales de mes las tropas navarras habían llegado a los límites de Vizcaya donde se detuvo la ofensiva hasta marzo de 1937.

1. Este artículo es una síntesis de mi estudio Violencia politica y represión en Guipuizcoa durante la Guerra Civil y el Primer Franquismo (1936-1945), San Sebastián, Hiria Liburuak, 2005.

2. Sobre la vida política y social en Guipúzcoa cfr. Rodríguez Ranz, José Antonio, Guipúzcoa y San Sebastián en las elecciones de la II República, San Sebastián, Fundación Kutxa, 1994 y BarRuso Barés, Pedro, El movimiento obrero en Guipúzcoa durante la II República. Organizaciones obreras y dinámica sindical, San Sebastián, Diputación Foral de Gipuzkoa, 1996. 
El fracaso de la sublevación en San Sebastián ${ }^{3}$ dio paso a la creación de un poder revolucionario, encarnado por la Junta de Defensa de Guipúzcoa y presidida por el diputado socialista Miguel de Amilibia, en el que se integraron todos los partidos que permanecieron fieles a la legalidad republicana incluidos los nacionalistas. La derrota de la sublevación dio paso a un período de control revolucionario de la provincia que no se tradujo en una violencia indiscriminada contra los derechistas ni desató una ola de violencia anticlerical. A pesar de ello, como veremos, sí se produjeron una serie de episodios de violencia y cinco sacerdotes perdieron la vida durante el período de control republicano de la provincia. Tras la ocupación de la capital, los sublevados pusieron en marcha una serie de procesos represivos que se prolongaron a lo largo de toda la Guerra Civil, si bien fueron perdiendo intensidad a medida que el conflicto se alejaba del territorio guipuzcoano lo cual no impidió que se produjera un repunte represivo al final de la contienda como consecuencia de la aprobación de la Ley de Responsabilidades Políticas y de la detención en otros lugares de personas que fueron acusadas de hechos ocurridos en Guipúzcoa durante la contienda.

Con estos puntos de partida, en las páginas siguiente nos planteamos realizar un análisis de la violencia política y de la represión que se llevó a cabo en Guipúzcoa partiendo de una serie de premisas básicas. La primera es la necesidad de abordar la represión en todas sus formas, de modo que podamos poner de manifiesto y analizar las interrelaciones entre ellas. Del mismo modo tratamos de ofrecer una visión global de todo el proceso, analizando tanto la violencia llevada a cabo por los republicanos como los procesos represivos puestos en marcha por los franquistas. Trataremos de esbozar las líneas maestras de los procesos represivos y poner de manifiesto cuáles fueron los sectores más afectados por los mismos, tanto desde una perspectiva espacial como política y social. Para llevar a cabo estos objetivos hemos partido de una muestra de más de seis mil represaliados, de los cuales tan sólo en torno al 10\% corresponden a la violencia ejercida por los republicanos (cfr. Cuadro 1), dada la imposibilidad de ofrecer cifras absolutas, pero que consideramos suficientes para que nos permita obtener unos resultados fiables de lo que supusieron los procesos represivos en Guipúzcoa entre 1936 y 1945.

\section{Cuadro 1. Procesados por las diversas jurisdicciones ${ }^{4}$}

\begin{tabular}{|l|c|c|}
\hline \multicolumn{1}{|c|}{ Jurisdicción Aplicada } & Casos & \% del Total \\
\hline Represión Económica & 1.841 & 29,89 \\
\hline Justicia Militar & 1.345 & 21,83 \\
\hline Depuración de la Administración & 1.330 & 21,59 \\
\hline
\end{tabular}

3. Sobre el desarrollo de la Guerra Civil en la provincia cfr. Barruso Barés, Pedro, Verano y revolución. La Guerra Civil en Gipuzkoa, San Sebastián, R\&B, 1996.

4. Sombreados los resultados de las jurisdicciones especiales de responsabilidad de los republicanos. 
De los tribunales populares a las comisiones depuradoras. Violencia y represión...

\begin{tabular}{|l|r|r|}
\hline Depuración de la Enseñanza & $\mathbf{7 2 8}$ & 11,82 \\
\hline Causa General & $\mathbf{3 8 3}$ & $\mathbf{6 , 2 2}$ \\
\hline Clero & 204 & 3,31 \\
\hline Tribunal Popular & $\mathbf{2 0 0}$ & $\mathbf{3 , 2 5}$ \\
\hline Masonería y Comunismo & 81 & 1,31 \\
\hline Prisioneros & 48 & 0,78 \\
\hline Total & $\mathbf{6 . 1 6 0}$ & $\mathbf{1 0 0}$ \\
\hline
\end{tabular}

Desde el punto de vista estrictamente cuantitativo, y como se puede apreciar en el cuadro 1, fue la represión económica el proceso que tuvo una mayor repercusión en la provincia, con casi el $30 \%$ de los casos analizados, seguida de la jurisdicción militar y las depuraciones laborales, que si las contemplamos de manera conjunta -Administración y Enseñanza- suponen el 33\% de los casos de represaliados en Guipúzcoa mientras que el resto de los procesos quedan a distancia de los anteriores. Por esta razón, en las páginas siguientes vamos a centrar nuestra atención, en primer lugar, en la violencia ejercida por los republicanos para, posteriormente, analizar los procesos represivos puestos en marcha por el franquismo.

\section{DE LA "VIOLENCIA ESPONTÁNEA» AL TRIBUNAL POPULAR}

El primer objeto de nuestro análisis debe ser necesariamente la violencia ejercida por los defensores de la República contra los sublevados, la cual presenta una serie de rasgos que es necesario destacar. En primer lugar debemos señalar que la violencia republicana es una realidad cambiante que evoluciona con rapidez pese al escaso tiempo que Guipúzcoa permaneció en manos de los republicanos. La primera manifestación de la violencia republicana, que podemos considerar como "violencia espontánea», se caracterizó por no responder a ningún planteamiento previo pese a que es apreciable la incidencia política de determinados grupos -en el caso de Guipúzcoa el PCE- en la que no se observa ningún tipo de formalidades jurídicas, las víctimas carecen por completo de medios de defensa y suele ser protagonizada por grupos más o menos organizados, y se salda con la muerte de un elevado número de opositores asesinados de manera sumaria.

El principal episodio de "violencia espontánea" -y el más importante- se produjo el 30 de julio de 1936 en el asalto a la prisión provincial en el que fueron fusilados medio centenar de presos derechistas que permanecían recluidos en la misma. Pese a que no fue, sin embargo, el único episodio que podemos considerar relacionado con la "violencia espontánea» ejercida por los republicanos sí que provocó que las organizaciones nacionalistas hicieran pública su protesta por el asesinato de los presos en una actitud inédita en la España republicana del verano de 1936.

La primera mutación que sufrió la violencia ejercida por los republicanos fue consecuencia de la creación del nuevo poder revolucionario que se creó en Guipúzcoa y que puso en marcha lo que hemos venido a denominar «justicia 
revolucionaria». Ésta se caracterizó por la dureza de la misma ya que fue, con excepción de la saca de presos anteriormente mencionada, la que mayor número de víctimas provocó entre las filas de los partidarios de la sublevación. A diferencia de la anterior sí es posible encontrar cierta organización y los procesados cuentan con ciertas garantías, al menos formales, pese a que se trata de una justicia rápida y sumaria y en gran parte concebida como represalia. La "justicia revolucionaria» nació como producto de la situación que se vivió en Guipúzcoa entre julio y septiembre de 1936, cuando el territorio guipuzcoano se había convertido en campo de batalla y las tropas de Mola provenientes de Navarra amenazaban con cerrar la frontera de Irún, lo que privaría a los republicanos de un punto vital para el suministro del norte aislado del resto de la zona republicana. Los bombardeos aéreos y navales sobre la costa guipuzcoana y San Sebastián se sitúan en el origen de la decisión de la Junta de Defensa de Guipúzcoa de poner en marcha una política de toma de rehenes con la finalidad de tratar de frenar los continuos bombardeos. De este modo la Junta de Defensa de Irún amenazó con juzgar y ejecutar a los rehenes más destacados que estaban en su poder entre los que se encontraba el mismísimo conde de Romanones, detenido en San Sebastián al comenzar la Guerra Civil. Por su parte, en la capital, y como consecuencia de sendos bombardeos navales, la Junta de Defensa adoptó la medida de celebrar dos consejos de guerra en los que fueron juzgados los principales militares implicados en la sublevación y que habían sobrevivido al asalto del 30 de julio. El primero de los consejos de guerra estuvo presidido por el comisario de guerra, y dirigente del PC de Euzkadi, Jesús Larrañaga y se saldó con la condena a muerte y ejecución de todos los procesados pese a los esfuerzos para evitar las ejecuciones del diputado nacionalista Manuel de Irujo y del embajador de Francia Jean Herbette ${ }^{5}$.

La tercera manifestación de la violencia protagonizada por los republicanos fue la que he denominado "justicia popular» y cuyo principal agente fueron los tribunales populares. Éstos, creados como tribunales de urgencia a finales de agosto de 1936, se ocuparon principalmente de las causas relacionadas con la sublevación. En Guipúzcoa, en función de los datos de que disponemos, podemos afirmar que llegó a crearse un tribunal popular, responsable de la condena a muerte de varios derechistas -entre ellos Víctor Pradera- en los días previos a la evacuación de San Sebastián por los republicanos. El resto de los presos derechistas fueron embarcados y trasladados a Bilbao donde fueron juzgados un elevado número de procesados guipuzcoanos tras la creación en la capital vizcaína del Tribunal Popular de Euzkadi.

Una de las características del Tribunal Popular de Euzkadi, en lo que se refiere a los procesados guipuzcoanos, fue su moderación. Pese a que se produjeron

5. Sobre la actuación del embajador francés cfr. Barruso Barés, Pedro, El frente silencioso. La Guerra Civil española en el Sudoeste de Francia (1936-1940), Alegia, Hiria Liburuak, 2001 y del mismo autor "La misión diplomática del embajador francés Jean Herbette durante la Guerra Civil", Bulletin d'Histoire Contemporaine de l'Espagne, n. ${ }^{\circ} 28-29$, décembre 1998-juin 1999, Aix en Provence, 2001, pp. 120-134. 
numerosas condenas a muerte en la mayor parte de los casos éstas recayeron sobre procesados ausentes con lo cual no tenían la menor repercusión. De los acusados, 19 condenados a muerte y ejecutados por el Tribunal Popular, sólo uno de los condenados - el teniente Lucio Vallespín- familiar directo del teniente coronel Vallespín, principal responsable de la conspiración en San Sebastián, era guipuzcoano mientras que el resto de los ejecutados lo fueron por hechos acontecidos en Vizcaya.

La conclusión final es que la represión republicana se fue moderando a medida que transcurría el tiempo, algo que es común a toda la zona republicana. Ésta experimentó su momento álgido en el verano de 1936 dando paso a una mayor moderación a medida que las autoridades republicanas y el Gobierno vasco se fueron haciendo con el control de la situación pero, para cuando esto ocurrió, la mayor parte de Guipúzcoa estaba en manos de los sublevados y la violencia republicana se había mostrado con toda su dureza en territorio guipuzcoano.

\section{LOS INICIOS DE LA REPRESIÓN FRANQUISTA: LA JUSTICIA MILITAR}

De manera simultánea a la ocupación de San Sebastián dio comienzo un proceso represivo protagonizado por las autoridades militares que se extendió desde el mes de septiembre al de noviembre de 1936, período en el que se produjeron un elevado número de ejecuciones sumarias. De manera paralela hubo una serie de episodios de violencia incontrolada por parte de los sublevados, como fue el fusilamiento de varios vecinos de Beasain el 28 de julio y que coincidió con la rendición de los sublevados que estaban sitiados en los cuarteles de la capital guipuzcoana. Aunque es difícil establecer una relación causa-efecto entre ambos acontecimientos sí que es cierto que cuando la resistencia republicana era intensa, como fue el caso de Beasain y luego de Tolosa e Irún, el número de ejecuciones sobre el terreno era mayor. Pero, sin lugar a dudas, en este primer período de la justicia militar en Guipúzcoa el hecho más determinante fue el fusilamiento de trece sacerdotes por parte de los sublevados.

La reciente publicación de documentos del archivo del cardenal Gomá y el estudio sobre el cardenal Segura ${ }^{7}$ han arrojado luz sobre la cuestión del clero vasco poniendo de manifiesto cómo los tradicionalistas, con su líder Fal Conde al frente, estaban dispuestos a aplicar, hasta las últimas consecuencias, el bando de declaración de estado de guerra del general Mola. Las ejecuciones de los sacerdotes se produjeron en unas circunstancias excepcionales y al margen de

6. Andrés-Gallego, José y Pazos, Antón María, Archivo Gomá: documentos de la guerra civi, Madrid, Consejo Superior de Investigaciones Científicas, 2002, vol. 2, enero de 1937 / con la colaboración de Santiago María Barroso; vol. 3, febrero de 1937 / con la colaboración de Santiago María Barroso Sánchez-Lafuente; vol. 4, marzo de 1937 / con la colaboración de Santiago María Barroso Sánchez-Lafuente; vol. 5, abril-mayo de 1937 / con la colaboración de Joaquín María Usunariz Belber.

7. Martínez SÁnchez, Santiago, Los papeles perdidos del cardenal Segura, 1880-1957, Pamplona, Eunsa, 2004. 
la «legalidad» que ya se estaba aplicando en Guipúzcoa. Mientras se estaban celebrando consejos de guerra con todas las formalidades los sacerdotes fueron víctimas de "sacas» como fue el caso de José Ariztimuno "Aitzol», fusilado junto con la mayor parte de los pasajeros del buque "Galerna» en el que fue capturado ${ }^{8}$, los clérigos de Mondragón Joaquín Arín, José Markiegi y Leonardo Guridi ejecutados el 25 de octubre de 1936 o Celestino Onaindía, hermano del que luego sería negociador del «Pacto de Santoña», Alberto Onaindía. Pero las ejecuciones de sacerdotes se detuvieron bruscamente tras un complejo proceso en el que intervinieron Gomá y el propio Franco pero que no pudo evitar la muerte del último sacerdote ejecutado, Jorge Iturricastillo, el 6 de noviembre de 1936 en circunstancias poco claras en las que posiblemente desde Burgos no se atendió la orden de Franco de detener las ejecuciones de sacerdotes.

Pero la represión militar en Guipúzcoa tuvo otras vertientes además de la antes relacionada. En líneas generales podemos decir que tras la dureza de los meses iniciales la situación remitió y descendió de manera notable el número de ejecuciones, no así el número de condenas a muerte, que siguió siendo elevado como demuestra la actuación posterior de la Comisión Provincial de Examen de Penas. Ésta, creada en enero de 1940, revisó 154 condenas a muerte que previamente habían sido conmutadas por treinta años. Tras el análisis de la documentación de la Comisión Provincial hemos podido documentar 639 casos de condenados a muerte en Guipúzcoa de los cuales 485, es decir el $76 \%$ de las mismas, fueron cumplidas principalmente entre 1936 y 1938 siendo éstos los años más duros de la represión militar. Sin embargo, debemos decir que si bien desde el punto de vista cuantitativo el número de ejecuciones disminuyó en la inmediata posguerra aumentó, de manera significativa, la vertiente cualitativa de la represión militar que se cobró las vidas, entre otros, del Gobernador Civil de Guipúzcoa, Antonio Ortega -fusilado en Alicante en 1939-, del concejal socialista de San Sebastián Luís Iglesias, fusilado en la capital donostiarra en 1940, o el comunista Jesús Larrañaga fusilado en Madrid en 1942.

Pero hay otros aspectos destacados en la represión ejercida por las autoridades militares en Guipúzcoa. Uno, y posiblemente de los más llamativos, es que el $53 \%$ de las denuncias presentadas -origen de la mayor parte de las causas militares analizadas-provienen de vecinos de los denunciados (33\%) o de familiares de las víctimas de la represión republicana (20\%) lo que nos da idea de la importante implicación de la población guipuzcoana en el proceso represivo. Por el contrario tan sólo en torno al $24 \%$ de las denuncias provienen de las autoridades (poder local 12\%, militares 7\%, funcionarios de la Administración 3\% y Guardia Civil 1,75\%). El clero, uno de los sectores que tradicionalmente ha sido considerado como implicado en la represión sólo dio origen en Guipúzcoa a un 1,75\% de las denuncias, un porcentaje similar al de la Guardia Civil. Finalmente decir que el perfil del procesado en Guipúzcoa por las autoridades militares corresponde al de un hombre que reside en la "Guipúzcoa Urbana» (en la

8. Para un mayor detalle de la captura del "Galema” cfr. BARRUSO BARÉs, Pedro, El frente silencioso... 
que se encuentran los principales municipios como Éibar, Rentería, Mondragón - Tolosa) ocupado en el sector secundario y que pertenecía a organizaciones de izquierda, principalmente la UGT aunque hay un elevado componente de nacionalistas, ideología a la que pertenecen la mayor parte de los procesados en la "Guipúzcoa Intermedia" y en la zona rural.

\section{LA CARA OCULTA DE LA REPRESIÓN: LA REPRESIÓN ECONÓMICA}

Tradicionalmente los estudios sobre la represión han centrado su atención de manera fundamental en el estudio de la justicia militar, la cual pese a ser muy importante, no fue la única jurisdicción represiva que se aplicó a las personas que el Nuevo Estado consideró como desafectas. Una de ellas, y que en Guipúzcoa tuvo un efecto que hizo que la considere como el más importante de los procesos represivos que se dieron entre 1936 y 1945, fue la represión económica que en el caso que ahora nos ocupa, y en cifras absolutas, supuso que más de cuatro mil personas se vieran implicadas en procesos represivos de tipo económico.

La represión económica dio comienzo muy pronto en Guipúzcoa y ya en septiembre de 1936 podemos documentar los primeros procesos de incautación de bienes. Sin embargo, la situación evolucionó rápidamente a partir de enero de 1937 tras la creación de la Comisión Provincial de Incautación de Bienes (CPIB), que entre 1937 y 1939 expedientó a más de 2.700 personas entre los que se encontraban los principales dirigentes políticos como era el caso del primer presidente del Gobierno vasco, José Antonio Aguirre, al que se le impuso en 1938 una sanción de 20.000 pesetas 9 .

Sin embargo fue la Ley de Responsabilidades Políticas, el principal instrumento represor del franquismo en Guipúzcoa desde el punto de vista económico. Pero ello no supuso que el proceso de aplicación y desarrollo de la LRP en Guipúzcoa fuese fácil ni arrojase los resultados que las autoridades franquistas esperaban. En primer lugar resultó complicada la transición de la responsabilidad de la represión económica de la CPIB a los Juzgados de Instrucción de Responsabilidades Políticas en parte por la ingente burocracia que se generó y en parte por la resistencia de algunas autoridades a poner en marcha la nueva jurisdicción, como quedó de manifiesto en las diferencias que se generaron entras las diferentes autoridades.

El roce más importante se produjo entre el juez instructor de responsabilidades políticas Flavio Lascaris -antiguo juez militar en Ciudad Real- y el gobernador civil Francisco Rivas y que arranca -desde mi punto de vista- en la percepción que se tenía en la provincia de que la represión era algo -que en su parte más dura- ya había pasado en Guipúzcoa. Sin embargo el juez consiguió imponer su opinión y una intensa actividad que se tradujo en la incoación de 1.650 expedientes de responsabilidades políticas entre 1939 y 1940 , que en

9. Sobre el caso del lehendakari José Antonio Aguirre, cfr. Barruso Barfs, Pedro, uJosé Antonio Aguirre y la justicia franquista", Sancho el Sabio, n. ${ }^{\circ} 18$ (2003), Vitoria, pp. 175-206. 
junio de 1943 eran ya 1.898 , mientras que casi doce mil esperaban para ser incoados.

Sólo la reforma de la ley en 1942 impidió que la aplicación de la misma tuviera efectos devastadores. Entre 1939 y 1942 el 75\% de los procesados habían sido condenados a penas económicas que oscilaban entre la pérdida total de bienes, como se produce en catorce ocasiones en Guipúzcoa, a sanciones menores pero que en algunos casos recaían sobre personas que habían sido fusiladas previamente y eran las familias las que debían hacer frente a la sanción. La represión ejercida mediante la aplicación de la Ley de Responsabilidades Políticas recayó, de manera fundamental, sobre la burguesía guipuzcoana, ligada a los intereses económicos y cercana al nacionalismo, como demuestra que el $31 \%$ de los expedientados en Guipúzcoa fueran acusados del cargo C (haber militado en alguno de los partidos ilegalizados por el decreto 108 de la Junta de Defensa Nacional) y un $19 \%$ lo fueron por el cargo L; atribuido a aquellas personas que se habían "opuesto de manera activa al Alzamiento Nacional».

Desde el punto de vista de la militancia política los nacionalistas fueron el grupo más afectado, ya que el $56 \%$ de los procesados son considerados como tales, frente a un $41 \%$ de izquierdistas. Dentro del nacionalismo el principal grupo de expedientados lo formaron los militantes del PNV seguidos a considerable distancia por los integrantes del resto de las organizaciones del entramado nacionalista. En lo que se refiere a la izquierda el grupo más castigado fue la UGT, pese a que la ley no contemplaba la posibilidad de expedientar a los simples militantes de las organizaciones obreras, seguida de Izquierda Republicana ${ }^{10}$. En lo que se refiere a la actividad económica de los procesados podemos decir que el $46 \%$ de los mismos pertenecían al sector terciario, fundamentalmente aquellos que son considerados como «empleados», seguido del sector secundario con un $38 \%$ de procesados y del primario con un $18 \%$. Sin embargo, de manera absoluta, el grupo más castigado es, nuevamente, el de los «obreros industriales", grupo al que pertenecen el $20 \%$ de los procesados. Quizá pueda sorprender este dato, si se tiene en cuenta que, como hemos dicho antes, el principal damnificado por la aplicación de la LRP fue la burguesía guipuzcoana. Maticemos esto. El supuesto A de la LRP facultaba a que aquellos que hubieran sido condenados por las autoridades militares fueran expedientados de manera automática por la mencionada jurisdicción. Dado que el grupo más castigado por las autoridades militares fue el de los obreros industriales, y que un $16 \%$ de los casos que hemos analizados habían sido previamente condenados en un consejo de guerra, no nos debe extrañar, por tanto, esta elevada presencia de los mismos.

A raíz de la reforma de 1942 todo cambió. La mayor parte de los expedientes que se instruyeron fueron sobreseídos y entre 1943 y 1945 sólo se dictaron

10. Sobre la represión de los republicanos guipuzcoanos cfr. BarRuso Barés, Pedro, "La represión del republicanismo en Guipúzcoa, 1936-1945», Cuadernos Republicanos, n. 38 (1999), Madrid, pp. $65-94$. 
15 sentencias condenatorias en Guipúzcoa. La reforma, forzada por el «atasco» generado en la Administración de Justicia más que por la clemencia del régimen, supuso la práctica liquidación de la jurisdicción derogada en 1945 aunque en no pocos casos los expedientes se prolongaron hasta el decreto de indulto de 1966. En resumen podemos decir que la LRP vino, en cierto modo, a "socializar" la represión. Ya no era un grupo determinado de personas quienes podían ser expedientadas, era la mayor parte de la sociedad. La amplitud de las actuaciones que considera delictivas la LRP hizo que el abanico de personas que podían verse implicadas se incrementase de una manera sustancial.

Uno de los aspectos que permite analizar la LRP es conocer qué pasó con los refugiados que volvieron a España, tratando de aclarar un aspecto poco estudiado en la historiografía del franquismo. Con la excepción de aquellos dirigentes - activistas políticos destacados que o bien fueron entregados a Franco como es el caso, entre otros, del presidente de la Generalitat, Lluís Compayns, o el director de "El Socialista" y ministro de Gobemación, Julián Zugazagoitia, ambos ejecutados, o dirigentes comunistas, como es el caso del "Grupo Lisboa» que corrieron la misma suerte que los anteriores pero poco sabemos de la suerte de los exiliados que regresaron a España. El análisis de los expedientes de responsabilidades políticas nos sitúan ante personas corrientes a las que la incertidumbre creada por el conflicto llevó al exilio. No pocos de ellos, cuando quisieron regresar, se toparon con la gran cantidad de condicionantes impuestos por las nuevas autoridades, lo que forzó a muchos de ellos a permanecer en Francia. En la mayor parte de los casos no se trataba de personas con implicación política y pese al importante regreso que se produjo tras la caída de Irún ha quedado demostrado que fueron las propias normas franquistas las que frustraron un gran número de regresos. Este retraso, motivado por la constante demanda de avales, se convirtió en la base de la acusación contra muchos refugiados cuando finalmente regresaron a España tras la ocupación de Francia por los alemanes. Muchos de ellos huyeron ante la posibilidad de que las autoridades alemanas optaran por su deportación, su enrolamiento en el Servicio de Trabajo Obligatorio o, como ocurrió en el caso de los dirigentes nacionalistas, ser confinados en el campo de Gurs. La sola permanencia en el extranjero se convirtió en causa de responsabilidad para un $8 \%$ del total de los expedientados que hemos analizado de los cuales la mayor parte regresaron en 1940, pensando que mejor sería una dictadura en España que permanecer en Francia ante un futuro incierto y dependientes de las autoridades alemanas.

La conclusión final que podemos extraer de la aplicación de la LRP en Guipúzcoa es que, pese a ser el principal elemento represor del franquismo en el territorio guipuzcoano, ésta se saldó con un fracaso. En el momento de producirse la reforma de la jurisdicción, en 1942, que en el caso de Guipúzcoa y a la vista de los resultados, casi supuso la liquidación de la misma, tan sólo se habían incoado 1.650 expedientes, de los cuales solo 534 se habían terminado. Es decir, muy lejos de los 12.000 expedientes que estaba previsto incoar. La complejidad de la incoación de los expedientes, la carencia de medios en los juzgados instructores y el desinterés de las autoridades que se debían ver implicadas en el 
proceso de instrucción de los expedientes hicieron que el desarrollo de la jurisdicción especial fracase. No podemos concretar, pero podemos suponer, que del mismo modo la aplicación de las sanciones se saldó con un fracaso.

Como ha quedado demostrado la finalidad de la Ley de Responsabilidades Políticas fue fundamentalmente económica. Si las otras jurisdicciones buscaban el encarcelamiento o la eliminación física del opositor más comprometido, mediante las jurisdicciones civiles se alcanzaba a un amplio sector de la población que podía haber quedado al margen de la justicia militar debido a que su actuación política no pasó de los comportamientos estrictamente normales en una sociedad democrática. La Ley de Responsabilidades Políticas además de sancionar a las personas que podían ser consideradas como "desafectas", las desarmaba económicamente, bien mediante la imposición de sanciones -que en no pocos casos complicarían las economías familiares en un período de crisis como fue la posguerra española- o mediante el despojo de todos su bienes en caso de encontrarse en el exilio o no poder hacer frente a la sanción el expedientado. De esta manera el Estado pasó a ser propietario de un gran número de inmuebles, muchos de los cuales luego pasaron a albergar dependencias oficiales o fueron vendidos a precios muy ventajosos a los partidarios del Nuevo Estado.

\section{LA DEPURACIÓN DE LA SOCIEDAD GUIPUZCOANA}

Posiblemente uno de los empeños del franquismo fue lograr una sociedad completamente afecta al mismo o, en su defecto, lograr apartar de los puestos de responsabilidad a aquellas personas que podían ser contrarias al mismo logrando, de esta manera, un control absoluto de los resortes del Estado. Del mismo modo, mediante la provisión de los puestos de trabajo que quedaban vacantes, muchos de ellos no necesariamente de responsabilidad política sino administrativa, se pudo ir generando un "clientelismo» entre las personas que ocupaban los cargos y su debida "gratitud" hacia el nuevo régimen a la vez que éste le «recompensaba» por sus servicios, logrando de este modo una Administración completamente «afecta». Pero, para ello, era necesario llevar a cabo una completa y compleja depuración de la sociedad española.

En el momento actual las depuraciónes quizá sea uno de los aspectos menos conocidos de la represión franquista aunque en el caso del Magisterio hemos experimentado un considerable avance en los últimos años de la mano de estudios regionales o provinciales. Pero junto al caso del Magisterio existe un amplio proceso depurador que nos es, por el momento, casi desconocido. En este aspecto Guipúzcoa no era una excepción. Salvo la primera aproximación al caso del Magisterio realizada por Maitane Ostolaza ${ }^{11}$ la cuestión de la depuración estaba sin tratar en lo que se refiere a Guipúzcoa.

En nuestro estudio nos hemos centrado en dos sectores de gran importancia por el número de personas que emplean; la Administración, tanto provincial

11. Ostolaza Esnal, Maitane, El garrote de la depuración. Maestros vascos en la Guerra Civil y el Primer Franquismo (1936-1945), San Sebastián, Ibaeta Pedagogía, 1996. 
como local, y al conjunto de la Enseñanza. Somos conscientes que quedan fuera de este análisis importantes sectores, en los que nos consta que se produjeron procesos depurativos, como es el caso de los colegios profesionales, pero la desaparición de la documentación o la negativa a acceder a la misma han hecho que estos sectores sean una de las cuestiones pendientes en lo que al estudio de la represión en Guipúzcoa se refiere.

Pero, centrándonos ya en el primero de los sectores, el de la Administración diremos que nuestro conocimiento es desigual. Si bien conocemos con detalle algunos procesos de depuración en ayuntamientos como es el caso de Andoain, Irún o Fuenterrabía ${ }^{12}$, nuestro conocimiento es más escaso en otros lugares.

De los más de 1.300 casos de funcionarios depurados que hemos analizado la mayor parte de ellos corresponden a la Diputación Provincial de Guipúzcoa y al Ayuntamiento de San Sebastián mientras que el resto corresponden a diversos ayuntamientos. Más de trescientos funcionarios de la Diputación de Guipúzcoa y el 50\% del personal del Ayuntamiento de San Sebastián fueron separados inicialmente del servicio al no reincorporarse a sus puestos de trabajo en el plazo indicado si bien nos consta que algunos de los empleados cesados fueron readmitidos posteriormente y algunos vieron como su reingreso estaba condicionado a su comportamiento en el frente de batalla integrados en el ejército franquista.

El proceso de depuración que conocemos con mayor detalle es el de los empleados municipales de Irún. El nuevo ayuntamiento, constituido tras la ocupación de la localidad, concedió dos días para que los funcionarios se reintegrasen a sus puestos de trabajo si estaban en la localidad y cinco si no se encontraban en la misma. Durante los meses de septiembre y octubre de 1936 más de cien funcionarios regresaron pero, dada la complejidad del proceso depurador, a finales de octubre de 1936 tan sólo cuarenta y nueve se habían reincorporado a sus puestos de trabajo. El proceso depurador tomó un nuevo rumbo en diciembre de 1936, como consecuencia del decreto de 5 de diciembre de 1936 de la Junta Técnica. Según la citada norma todos los funcionarios que no habían regresado debían ser cesados definitivamente y al resto se les abría expediente de depuración, dando comienzo a un nuevo proceso que en el caso de Guipúzcoa coincide con una campaña contra el nacionalismo y contra todas aquellas personas que podían ser sospechosas de haber pertenecido a organizaciones nacionalistas a la vez que desde la prensa de San Sebastián se pedía mano dura para con «los enemigos de España» señalando que «el que ha gritado Muera España no merece pisar la tierra de la Patria» ${ }^{13}$.

12. Para una referencia más detallada al proceso seguido en los mencionados municipios cfr. BARRUso BAREs, Pedro, «Poder político y representación social en Andoain durante el Primer Franquismo", Leyçaur, n. ${ }^{\circ} 6$ (2000), Andoain, 2000, pp. 255-350; Destrucción de una ciudad y construcción de un Nuevo Estado. Irín en el Primer Franquismo (1936-1945), Irún, Archivo Municipal de Irún, 2003, en especial las páginas 120 a 130 y "República, Guerra y Franquismo (1936-1959)», en Historia de Hondarribia, Ayuntamiento de Hondarribia, Hondarribia, pp. 325-354.

13. El Diario Vasco, 18 de noviembre de 1936. 
En Irún, donde conocemos con mayor detalle el proceso depurador de la Administración, como consecuencia de la nueva norma se instruyeron sesenta expedientes de depuración. En la instrucción de los mismos se recogieron informes procedentes del propio ayuntamiento, de las fuerzas del orden, de las organizaciones políticas e, incluso, de particulares. Con ellos se iniciaba un largo proceso depurador que no se cerró hasta mediados de los años cuarenta cuando se fallan los últimos expedientes de depuración y que se saldó con un $45 \%$ de funcionarios sancionados con penas que van desde la separación del servicio, como ocurre en diecisietes casos, a sanciones menores de privación de empleo y sueldo durante un determinado tiempo.

El otro gran proceso depurador que conocemos en detalle es el de la Enseñanza. Como novedad más importante podemos señalar el estudio de la actuación de la llamada "Comisión C", encargada de la depuración de la Enseñanza Secundaria. De ese modo, y debido a que en Guipúzcoa no existía universidad ni escuelas de ingenieros - de cuya depuración se ocuparon, respectivamente, las Comisiones A y B-tenemos una panorámica completa de la depuración de la Enseñanza en el territorio guipuzcoano.

Desde un punto de vista estrictamente cuantitativo podemos decir que el resultado de la depuración ofrece resultados similares tanto en el caso de la Enseñanza Primaria como en la Secundaria. Si en Primaria son sancionados el $27 \%$ de los maestros, en Secundaria se sancionó al $26 \%$ del profesorado.

\section{Cuadro 2. Resultado de la depuración de la enseñanza en Guipúzcoa}

\begin{tabular}{|l|r|c|}
\hline \multicolumn{1}{|c|}{ Resultado de la depuración } & Primaria & Secundaria \\
\hline Confirmados & $70,70 \%$ & $73,58 \%$ \\
\hline Inhabilitados & 0 & $5,66 \%$ \\
\hline Sancionados & $14,46 \%$ & $5,66 \%$ \\
\hline Cesados & $14,68 \%$ & $15,09 \%$ \\
\hline Fallecidos & $3 \%$ & 0 \\
\hline Desconocido & $0,9 \%$ & 0 \\
\hline
\end{tabular}

Si relacionamos estas cifras con las de otras provincias en las que conocemos el resultado del proceso depurador observaremos, en lo que a Primaria se refiere, que tan sólo provincias como Asturias, Barcelona o Girona ofrecen cifras superiores a las del caso guipuzcoano. Esto nos permite afirmar que estamos ante un proceso depurador de extremada dureza que se prolongó en el tiempo y que separó del servicio o sancionó a más de la cuarta parte de los educadores guipuzcoanos.

Del mismo modo que ocurre en el resto de los procesos represivos la depuración de la Enseñanza en Guipúzcoa tiene una serie de matices que debemos destacar. El primero de ellos es el escaso número de maestros fusilados. Tan sólo uno de ellos fue ejecutado, el maestro de Mondragón Ángel Iturmendi, socialista y procesado en la Revolución de Octubre de 1934 acusado de haber 
redactado el bando del comité revolucionario de dicha localidad. Iturmendi, sin embargo, no fue fusilado hasta 1938, tras ser detenido en Asturias a donde había huido siguiendo el curso de la Guerra Civil. Pero lo que no ofrece ninguna duda es que el principal objetivo de la depuración de la Enseñanza, al menos en el caso de Primaria, fue la erradicación del nacionalismo del ámbito escolar. Así se hizo constar en la circular del 7 de diciembre de 1936, de la Comisión de Cultura y Enseñanza de la Junta Técnica, en la que se señala la necesidad de trasladar a los maestros «que siendo profesional y moralmente intachables, hayan simpatizado con los titulados partidos nacionalista vasco, catalán, navarro, gallego etc..." mientras que para los maestros de izquierda se reservaba, pura y simplemente, la separación del cuerpo.

Pero la aplicación de esta norma en el caso de Guipúzcoa presentaba una serie de complicaciones. Por una parte los maestros considerados nacionalistas eran a su vez, dado su catolicismo, útiles en otras zonas de España donde no se hubiera desarrollado el nacionalismo. Del mismo modo, y pese a que se hace mucho hincapié en la eliminación de todo izquierdismo, librepensamiento, influencia de la Institución Libre de Enseñanza o del liberalismo en la escuela, se reconoce que determinados maestros que debían ser trasladados por las acusaciones que se formulaban contra ellos, era posible que permanecieran en el País Vasco al no ser nacionalistas y considerar que podrían realizar una labor importante en pro de la eliminación del nacionalismo de las escuelas.

En la práctica la depuración en Guipúzcoa se convirtió en un proceso en que se enjuiciaron los comportamientos políticos, profesionales y personales de los maestros. Los informes, a veces contradictorios, eran fundamentales a la hora de decidir la suerte de un maestro y en el que las informaciones procedentes del clero parroquial eran determinantes. Toda conducta que se apartase de la estricta ortodoxia católica era censurada y suponía un grave cargo para el maestro en cuestión y, posiblemente, le hacía acreedor de una sanción. Si a lo anterior se unía alguno de los otros cargos la suerte del maestro, profesionalmente hablando, se podía ver seriamente comprometida.

La depuración de la Enseñanza Secundaria tuvo algunos matices que la diferenciaron del proceso seguido en la primera enseñanza. En primer lugar la masa numérica, la quinta parte de la anterior, y en segundo la localización de los centros de enseñanza, situados en las principales localidades (San Sebastián, Eibar, Irún y Oñate). El proceso depurador es similar al caso del Magisterio. A la instrucción del expediente le sigue el recabar informes sobre el depurado para concluir, en su caso, en la formulación de un pliego de cargos al cual el expedientado podía replicar con el consabido pliego de descargos. Lo mismo que ocurre en el caso del Magisterio los cargos se pueden agrupar en cuatro grandes grupos (cargos políticos, sindicales, profesionales y morales) si bien en el caso de Secundaria son los dos primeros los más importantes. La pertenencia a las organizaciones de izquierda o nacionalistas estaba presente en la mayor parte de los expedientes en los que se formulan cargos. Pero también el componente religioso tiene un peso destacado. La calificaciones de "ateo", "católico tibio", "profesar doctrinas disolventes"... son frecuentes en los expedientes instruidos 
por la Comisión C pero, al contrario de los que se podía pensar, no aparecen en los informes de los clérigos, que no son preceptivos en este caso. Los informes procedentes de sacerdotes o párrocos, a diferencia de lo que ocurre en Primaria, son presentados como avales por los expedientados, confirmando la tesis de que gran parte del clero guipuzcoano se mantuvo al margen de los procesos represivos y, cuando tuvo que tomar parte en ellos, sus informaciones generalmente fueron -evidentemente con excepciones- favorables al expedientado. La explicación de esta actuación del clero guipuzcoano se puede buscar, en primer lugar, porque él mismo es objeto de la represión y, en segundo lugar, por la estructura del clero provincial en el que podemos encontrar que un importante sector se encuentra alejado de los planteamientos reaccionarios e integristas de otros sectores del clero ${ }^{14}$.

Completa el panorama represivo en Guipúzcoa la aplicación de la Ley de Represión de la Masonería y el Comunismo puesta en vigor en 1940. Ésta, pese a ser la jurisdicción especial con mayor duración temporal, ya que estuvo en vigencia hasta 1963, tuvo una escasa repercusión en Guipúzcoa. Los masones guipuzcoanos eran pocos y en algunos casos se trataba de personas que provenían de otras provincias lo cual no fue óbice para que el porcentaje de condenas fuese elevado y las penas de prisión elevadas al ser la mayoría de ellas de 12 años y 1 día de prisión.

\section{CONCLUSIÓN: UNA REPRESIÓN INTENSA DE UNA SOCIEDAD CONSERVADORA}

En conclusión podemos decir que la principal característica de la represión franquista en Guipúzcoa fue su intensidad y el amplio espectro social al que afectó. Calculamos que el $2 \%$ de la población, porcentaje que si lo extrapolamos a las familias de los procesados podría situarse en cifras entre el 8 y el $10 \%$ del total de la población, se vio afectada por procesos represivos. La represión en Guipúzcoa fue un proceso controlado en su casi totalidad por las autoridades militares, ya en los consejos de guerra, juzgados y tribunales de responsabilidades políticas y Tribunal de Responsabilidades Políticas, la mayoría de los miembros eran militares. Su influencia es menor en los procesos de depuración pero, en última instancia, las propuestas de las diversas comisiones, como es el caso del Magisterio, debían ser elevadas al Gobernador Civil, cargo que durante gran parte de la Guerra Civil recayó en un militar.

Estamos, por tanto, ante un proceso represivo de una intensidad y de una duración inusitada. La represión en Guipúzcoa, al igual que en otros lugares, que sin embargo consideramos que excedió todos los planes iniciales previstos en la conspiración. La represión puesta en marcha por los sublevados perseguía la eliminación de los adversarios, la eliminación de toda posibilidad de oposi-

14. Sobre el clero guipuzcoano $f f r$. ArzPuRU, Mikel y UnAnue, Donato, «El clero diocesano guipuzcoano y el nacionalismo vascon, en Beramendr, Justo G. y MAz, R. (Comps.), Los nacionalismos en la España de la II República, Madrid, Siglo XXI, 1991, pp. 287-304. 
ción organizada y la desmovilización de los posibles opositores al privarles de sus bienes o medios de vida, de modo que se viesen sumidos en la mera supervivencia. El proceso represivo que se llevó a cabo entre 1936 y 1945 supuso una época de una violencia desconocida hasta ese momento en Guipúzcoa. Las diversas jurisdicciones, pese a pretender actuar de manera coordinada, no lograron alcanzar los objetivos iniciales. Sin embargo la presión que se ejerció sobre la sociedad guipuzcoana fue suficiente para el número de las personas que se opusieron al franquismo de manera activa en los primeros años fuera muy reducido.

En lo respecta a la represión republicana ésta fue, de igual manera, de una violencia inusitada. En los apenas cincuenta y cinco días de control republicano de Guipúzcoa se produjeron casi cuatrocientas víctimas, lo que da idea de la magnitud del drama. A diferencia de lo que ocurre con la represión franquista las víctimas de la represión republicana pertenecían a un sector muy concreto de la sociedad, ligado a la monarquía o a las opciones derechistas y con importantes intereses económicos. A pesar de su breve período de vigencia, evolucionó rápidamente desde actuaciones de violencia incontrolada (asalto a las cárceles, sacas de presos) hasta un proceso de "institucionalización" por medio de la Junta de Defensa o los Tribunales Populares, jurisdicciones -en todo caso- excepcionales y al margen de la justicia ordinaria.

Finalmente considero necesario hacer una referencia al camino que queda por recorrer. En primer lugar es necesario profundizar en la jurisdicción militar, trascendiendo del estudio de los «anteriores» -es decir aquéllos cuyas causas están motivadas por hechos acontecidos durante la Guerra Civil- para adentrarse en el análisis de los casos de los "posteriores", aquellos que siguieron actuando políticamente en el franquismo y que en algunos casos podían haber jugado algún papel durante la Guerra Civil. Es necesario analizar, a medida que se vayan catalogando los documentos, el entramado económico organizado por el régimen al amparo de los batallones de trabajadores y las colonias penitenciarias militarizadas. El cruce de intereses políticos y económicos, con participación de grandes empresas y de la Dirección General de Regiones Devastadas, es una cuestión pendiente de la que vamos conociendo diversos aspectos mediante obras como la dedicada al Canal del Guadalquivir ${ }^{15}$. Del mismo modo es necesario proseguir el estudio de las depuraciones de colectivos concretos, generalmente profesiones liberales, para lo cual es imprescindible que se pueda acceder con libertad a los fondos de los colegios profesionales. Y, finalmente, queda el estudio de la violencia encubierta que a lo largo de muchos años privó a los tildados de "rojos» del acceso a determinados puestos de trabajo, a desempeñar determinadas responsabilidades o a ser excluidos socialmente por el mero hecho de ser considerados "desafectos" y que estigmatizó incluso a las

15. Acosta Bono, Gonzalo, Gutierrez Molina, José Luis, Martínez Macias, Lola y Rio Sánchez, Ángel del, El canal de los presos. Trabajos forzados: De la represión política a la explotación económica, Barcelona, Crítica, 2004. 
familias de los represaliados en un proceso que en ocasiones se prolongó hasta el final del franquismo. 